\title{
Output Feedback Finite-Time Stabilization of Systems Subject to Hölder Disturbances via Continuous Fractional Sliding Modes
}

\author{
Aldo-Jonathan Muñoz-Vázquez, ${ }^{1}$ Vicente Parra-Vega, ${ }^{2}$ \\ Anand Sánchez-Orta, ${ }^{2}$ and Gerardo Romero-Galván ${ }^{1}$ \\ ${ }^{1}$ Electrical and Electronic Engineering Department, Autonomous University of Tamaulipas, \\ Reynosa-Rodhe Campus, Reynosa, TAMPS, Mexico \\ ${ }^{2}$ Robotic and Advanced Manufacturing Department, Research Center for Advanced Studies, \\ Saltillo Campus, Ramos Arizpe, COAH, Mexico \\ Correspondence should be addressed to Aldo-Jonathan Muñoz-Vázquez; aldo.munoz.vazquez@gmail.com
}

Received 23 June 2017; Revised 16 August 2017; Accepted 29 August 2017; Published 8 October 2017

Academic Editor: Bogdan Dumitrescu

Copyright (C) 2017 Aldo-Jonathan Muñoz-Vázquez et al. This is an open access article distributed under the Creative Commons Attribution License, which permits unrestricted use, distribution, and reproduction in any medium, provided the original work is properly cited.

\begin{abstract}
The problem of designing a continuous control to guarantee finite-time tracking based on output feedback for a system subject to a Hölder disturbance has remained elusive. The main difficulty stems from the fact that such disturbance stands for a function that is continuous but not necessarily differentiable in any integer-order sense, yet it is fractional-order differentiable. This problem imposes a formidable challenge of practical interest in engineering because (i) it is common that only partial access to the state is available and, then, output feedback is needed; (ii) such disturbances are present in more realistic applications, suggesting a fractional-order controller; and (iii) continuous robust control is a must in several control applications. Consequently, these stringent requirements demand a sound mathematical framework for designing a solution to this control problem. To estimate the full state in finite-time, a high-order sliding mode-based differentiator is considered. Then, a continuous fractional differintegral sliding mode is proposed to reject Hölder disturbances, as well as for uncertainties and unmodeled dynamics. Finally, a homogeneous closed-loop system is enforced by means of a continuous nominal control, assuring finite-time convergence. Numerical simulations are presented to show the reliability of the proposed method.
\end{abstract}

\section{Introduction}

It is a standard practice to design systems with only one measurement, such that its derivatives are obtained with approximation schemes. In addition, robustness and continuous control have become common requirements. These stringent characteristics represent qualitative and quantitative advantages to be considered in high-end controllers, a fact that makes it necessary to design controllers with only partial access to the state. The requirements of continuity and robustness stand for a whole different problem when disturbance is Hölder continuous. The lack of integer-order differentiability of Hölder functions suggests the design of fractional-order control since such functions exhibit wellposed derivatives just for some fractional orders. In essence, the problem statement is defined as follows: design an output feedback stabilization scheme that converges in finite-time (the separation principle does not apply to nonlinear systems) with robust continuous (integral-type) sliding modes, designed with differintegral tools of fractional calculus. More precisely, we study this problem by assuming an integer-order plant given in regular form and subject to Hölder disturbances.

Let us analyze each dimension of the problem, in the realm of finite-time, output feedback, and fractional-order control. Finite-time stability is an appealing property due to fast convergence that comparatively shows improved performance against a wide class of dynamical uncertainties [1], inspired in terminal sliding modes [2], based on terminal attractors [3], and, using a continuous feedback that is non-Lipschitz around the equilibrium, pioneering 
applications in robotics were studied in [4-6]. In particular, the method of [2] is extended in $[1,7,8]$ for finite-time stabilization of second-order systems with a continuous feedback control, exploiting the key property of homogeneity [9] that preserves structural properties of the plant, such as scaling, dilation, or contraction mappings. The homogeneity concept is studied for continuous finite-time stabilization of a chain of integrators of $n$-order in [10], which has been explored for a smooth version of the supertwisting control in [11]. This provides a method to deal with output feedback with uncertainties [12]; however, it is discontinuous and asymptotic and then extended in [13] without disturbances, considering a model-based extended state observer. Nevertheless, all these schemes assume that, at most Lipschitz continuous disturbances, then, none of these apply to the case of Hölder disturbances. The main motivation for considering the case of Hölder disturbances is that on one hand the Hölder condition generalizes the Lipschitz condition to a larger class of functions without a first-order derivative but having fractional-order derivatives for all orders less than a critical order in turns less than one [14]; on the other hand, fractional-order derivatives allow us to consider more wider and realistic physical phenomena associated with fractionalorder models $[15,16]$ since fractional calculus provides a deeper understanding of the real nature $[17,18]$.

From the standpoint of engineering, fractional-order control has drawn the attention since it not only provides an alternate control method but also shows a rich frequency response; see the simple mechanical plants [19] up to quite advanced applications in aeronautics [20]. A restrictive class of nonlinear systems with a model-free output feedback and a model-based observer is studied in [21], while [22] studies the case of fractional parametric uncertainties and [23] studies singular linear fractional-order systems. In contrast, [24] shows finite-time stabilization with continuous integral sliding modes of a chain of integrators subject to Lipschitz disturbances; however, it applies only to disturbances that possess a bounded, at least weak, integer-order derivative, which fails for Hölder disturbances; in addition, access to the full state is assumed.

1.1. Proposed Solution and Contribution. The lack of integerorder differentiability of the disturbance and the continuous control requirement stand for a new class of control design that has remained elusive in the literature. We study finitetime state estimation and fractional-order finite-time stabilization of a class of dynamic systems subject to Hölder disturbances to produce an output feedback continuous fractional-order controller.

Firstly, a high-order sliding mode-based differentiator [9, 25 ] is considered for robust and finite-time exact estimation of the output and its derivatives. Secondly, recent results in [26-28] are adopted to design a fractional-order sliding mode control that rejects exactly (not just equivalently) the matched Hölder disturbances in finite-time, by invoking the topological properties of differintegral operators [17]. Finally, a nominal controller is designed to enforce finite-time convergence of the whole (pseudo)state [10]. To summarize, our proposal satisfies the problem statement by the following: (i) Finite-time stabilization with exact rejection of Hölder disturbances

(ii) The proposed controller is continuous, whose regularity establishes a compromise with respect to the critical exponent of the Hölder function

(iii) Finite-time output feedback control

1.2. Organization. Next section presents the fundamentals on fractional calculus and on Hölder functions. Section 3 shows the control design. Section 4 provides simulation results that highlight the reliability of the proposed method. Finally, main conclusions are given in Section 5.

\section{Preliminaries on Fractional Calculus}

2.1. Fractional Differintegral Operators. Fractional calculus is based on fractional-order integrodifferential operators, called differintegral operators. Consider the real-valued function $f: \Omega \rightarrow \mathbb{R}$, with $\Omega \subset \mathbb{R}$ a closed and bounded time-interval. The interested reader is referred to $[14,17,18,26-28]$, and for completeness, in this section, the differintegral operators and some properties of Hölder functions are introduced. Consider $\beta \in(0,1]$ and the following differintegral operators:

(i) Riemann-Liouville Fractional Integral

$$
{ }_{a} I_{t}^{\beta} f(t)=\frac{1}{\Gamma(\beta)} \int_{a}^{t} \frac{f(\tau)}{(t-\tau)^{1-\beta}} d \tau .
$$

(ii) Extended Caputo Fractional Derivative

$$
\begin{aligned}
{ }_{a}^{E} D_{t}^{\beta} f(t)= & \frac{f(t)-f(a)}{\Gamma(1-\beta)(t-a)^{\beta}} \\
& +\frac{\beta}{\Gamma(1-\beta)} \int_{a}^{t} \frac{f(t)-f(\tau)}{(t-\tau)^{\beta+1}} d \tau .
\end{aligned}
$$

(iii) Grünwald-Letnikov Integral

$$
{ }_{a} I_{t}^{\beta} f(t)=\lim _{T \rightarrow 0} T^{\beta} \sum_{k=0}^{\lfloor(t-a) / h\rfloor} w_{k}^{\beta} f(t-k h),
$$

where $\Gamma(x)=\int_{0}^{\infty} z^{x-1} e^{-z} d z$ is the Gamma function and $\lfloor\beta\rfloor=\max \{x \in \mathbb{Z}: x \leq \beta\}$. The coefficients $w_{k}^{\beta}$ are computed as $w_{k}^{\beta}=(1+(\beta-1) / k) w_{k-1}^{\beta}$, with $w_{0}^{\beta}=1$.

Note that, for $f(t)$ a differentiable function, integration by parts in (2) exactly renders the following classical fractional derivative.

(iv) Caputo Fractional Derivative

$$
{ }_{a}^{C} D_{t}^{\beta} f(t)={ }_{a} I_{t}^{1-\beta} \frac{d}{d t} f(t) .
$$

Moreover, notice that operator (4) is not suitable for studying the problem of Hölder disturbances due to a local definition is employed, and then (2) is considered instead. 
2.2. Hölder Functions and Fractional-Order Differentiability. Fractional-order operators provide tools to study nonlocal topological properties of continuous functions, such as their regularity degree in connection with the Hölder condition $[17,26]$. In addition, it has been shown that the fractionalorder integral of the discontinuous signum function preserves the main features required to design sliding mode control [29], including finite-time convergence and exact rejection of Hölder disturbances by means of a continuous signal of control.

In the sequel, let $\Omega=[a, b] \subset \mathbb{R}$ be a closed and bounded interval of nonzero Lebesgue measure, and then consider the following definition [17].

Definition 1. The real-valued function $\varphi: \Omega \rightarrow \mathbb{R}$ belongs to the Hölder space $\mathscr{H}^{\nu}(\Omega)$, with $v \in(0,1)$, if $\exists H \in \mathbb{R}$ constant, such that

$$
\left|\varphi\left(t_{2}\right)-\varphi\left(t_{1}\right)\right| \leq H\left|t_{2}-t_{1}\right|^{\nu}
$$

$\forall\left[t_{1}, t_{2}\right] \subseteq \Omega$, where $v$ is called the Hölder exponent of $\varphi$ in $\Omega$. The maximum $\nu$ such that $\varphi$ complies to the Hölder condition (5) is denoted as the critical exponent/order of $\varphi$.

The critical exponent $v_{c}$ of a continuous function provides a measure of its regularity. In case of $v_{c}=0$, such function is just bounded, but not necessarily continuous nor differentiable and for $v_{c}=1$ that function is Lipschitz continuous. Thus, the following propositions and lemmas are of interest.

Proposition 2. For $[\eta, \nu] \subseteq[0,1]$, one has that $\mathscr{H}^{\nu}(\Omega) \subseteq$ $\mathscr{H}^{\eta}(\Omega)$.

In addition, one has the following.

Lemma 3. $\mathscr{H}^{\nu}(\Omega)$ is a Banach space whose norm is

$$
\|\varphi\|_{\mathscr{H}^{\nu}(\Omega)}=\sup |\varphi|+[\varphi]_{\mathscr{C}^{\nu}(\Omega)},
$$

for $\varphi \in \mathscr{H}^{\nu}(\Omega)$, where $[\varphi(t)]_{\mathscr{H}^{\nu}(\Omega)}=\sup _{t_{1}, t_{2} \in \Omega}\left(\mid \varphi\left(t_{2}\right)-\right.$ $\left.\varphi\left(t_{1}\right)|/| t_{2}-\left.t_{1}\right|^{\nu}\right)$ provides to be a seminorm.

Proposition 4. Let $\varphi \in \mathscr{H}^{\nu}(\Omega)$ and $\psi \in \mathscr{H}^{\eta}(\Omega)$, where $\nu, \eta \in$ $(0,1)$. Then, $\varphi+\psi, \varphi \psi \in \mathscr{H}^{\min (\eta, v)}(\Omega)$. In addition, if $\varphi \in$ $\mathscr{H}^{\nu}(\psi[\Omega])$ and $\psi \in \mathscr{H}^{\eta}(\varphi[\Omega])$, then $\varphi \circ \psi, \psi \circ \varphi \in \mathscr{H}^{\nu \eta}(\Omega)$, for o the composition operator.

Notice that the algebraic properties of Hölder spaces defined in Proposition 2 and Corollary 2 of Lemma 3.1 of [17], p. 56, unveil the advantageous property that the fractionalorder integration improves the topological properties of a bounded function, as follows.

Lemma 5. The fractional integral $I_{t} I_{t}^{v}$ is a bounded operator from $\mathscr{H}^{0}(\Omega)$ into $\mathscr{H}^{v}(\Omega)$.

Lemma 6. Let $\varphi \in \mathscr{H}^{0}(\Omega)$; then ${ }_{a}^{E} D_{t}^{v}{ }_{a} I_{t}^{v} \varphi(t)=\varphi(t)$.
Lemma 7. Let $\varphi \in \mathscr{H}^{\lambda}(\Omega)$, with $\lambda \in(0,1]$. Then, $\forall v \in(0, \lambda)$, ${ }_{a}^{E} D_{t}^{v} \varphi(t)$ is continuous on $\Omega$. In addition ${ }_{a} I_{t}^{v}{ }_{a}^{E} D_{t}^{v} \varphi(t)=\varphi(t)-$ $\varphi(a)$.

Corollary 8. Let $\varphi \in \mathscr{H}^{\lambda}(\Omega)$, with $\lambda \in(0,1]$. Then, for $v \in$ $(0, \lambda), \sup _{t_{1}, t_{2}}\left|t_{t_{1}}^{E} D_{t}^{v} \varphi(t)\right|_{t=t_{2}} \leq[\varphi]_{\mathscr{H}^{v}(\Omega)}$.

In particular, the last two lemmas and the corollary are useful for stability analysis.

\section{Continuous Finite-Time Stabilization}

3.1. System Description and Control Statement. Consider the following class of dynamic systems:

$$
\begin{gathered}
\dot{x}_{1}=x_{2}, \\
\dot{x}_{2}=x_{3}, \\
\vdots \\
\dot{x}_{n}=u+\varphi, \\
y=x_{1},
\end{gathered}
$$

where $x=\left[x_{1}, x_{2}, \ldots, x_{n}\right]^{T} \in \mathbb{R}^{n}$ defines the pseudostate, for $x_{j}: \Omega \rightarrow \mathbb{R}, j \in\{1,2, \ldots, n\}$, with $\Omega$ the timeinterval at which the system is studied, output $y=x_{1}$ stands for the measured variable, $u$ is the control input, and $\varphi: \Omega \rightarrow \mathbb{R}$ represents matched disturbances and uncertainties. Examples of Hölder effects in physical systems modeled throughout $\varphi$, are given in [27-31].

Despite the fact that the system of (7) models a large class of plants of relative degree $d=n$, at first glance, it may seem a bit restrictive; however, it includes the large class of controllable nonlinear systems of the form $\dot{x}=f(x)+$ $g(x)(u+\varphi) \in \mathbb{R}^{n}$, with $f(x), g(x) \in \mathbb{R}^{n}$ smooth vector fields, and the case of linear time invariant systems with $f(x)=A x$ and $g(x)=B$, where $A \in \mathbb{R}^{n \times n}$ and $B \in \mathbb{R}^{n}$ are constant matrices, such that the pair $(A, B)$ is controllable. Clearly, for plants of relative degree $n$, and known flows, the system can be transformed into a chain of integrators of order $n$ by considering a function $y=h(x)$, with $L_{g} L_{f}^{i-1} h(x)=0 \forall i=$ $1, \ldots, n-1$ and $L_{g} L_{f}^{n-1} h(x) \neq 0$, which results in $y^{(n)}=$ $L_{f}^{n} h(x)+L_{g} L_{f}^{n-1} h(x)(u+\varphi)$, with $L_{f} h(x)=(\partial h / \partial x) f(x)$ the derivative of $h(x)$ along $f(x)$ [32]. Thus, system (7) models a large class of dynamical systems, including a vast set of physical systems. By example, let a system of $m$ degrees of freedom be modeled by the Euler-Lagrange formalism:

$$
H(q) \ddot{q}=\tau+f_{0}(t, q, \dot{q}),
$$

for $q \in \mathbb{R}^{m}$ the vector of generalized coordinates, $H(q) \in$ $\mathbb{R}^{m \times m}$ the positive definite inertia matrix, $\tau$ the control, and $f_{0}(t, q, \dot{q})$ the nonlinear terms as exogenous disturbances. Notice, however, that if such system is a mechanism, $f_{0}(t, q, \dot{q})$ includes friction, gravity, centripetal, centrifugal, and Coriolis forces; moreover, similar equations can be used to model some chemical process, electrical plants, and so 
forth. Now, considering $\mathbf{x}_{1}=q, \mathbf{x}_{2}=\dot{q}, \mathbf{u}=H^{-1}(q) \tau$, and $\varphi\left(t, \mathbf{x}_{1}, \mathbf{x}_{2}\right)=H^{-1}(q) f_{0}\left(t,\left(\mathbf{x}_{1}, \mathbf{x}_{2}\right)\right)$, then

$$
\begin{aligned}
& \dot{\mathbf{x}}_{1}=\mathbf{x}_{2}, \\
& \dot{\mathbf{x}}_{2}=\mathbf{u}+\varphi .
\end{aligned}
$$

This system stands for a perturbed second-order integrator, whose $i$ th component leads to the scalar perturbed secondorder integrator:

$$
\begin{aligned}
& \dot{x}_{1 i}=x_{2 i} \\
& \dot{x}_{2 i}=u_{i}+\varphi_{i} .
\end{aligned}
$$

In this way, there are a lot of dynamical plants described by perturbed chains of integrators.

Now, consider system (7) subject to an unknown Hölder disturbance $\varphi$ of critical order $v_{c} \in(0,1)$, and assume the partial state measurement only of $y=x_{1}$. Thus, we are ready to yield the control problem statement as follows:

Design a continuous controller $u: \Omega$

$\longrightarrow \mathbb{R}$, such that $x_{1}=0$

$$
\forall t \geq t_{f} \text {, for some } t_{f}<\infty .
$$

3.2. Output Feedback Robust Estimation. Considering access only to the output $y=x_{1}$, we aim at producing in finitetime their derivatives up to $n$-order by means of the exact robust differentiator of [9]. Thus, consider the following $n$ order differentiator, which is homogeneous of degree $r_{q}=-1$, [33]:

$$
\begin{gathered}
\dot{\hat{x}}_{1}=z_{1}+\widehat{x}_{2} ; \quad z_{1}=\lambda_{n-1}\left\lceil x_{1}-\hat{x}_{1}\right\rfloor^{(n-1) / n} \\
\dot{\hat{x}}_{2}=z_{2}+\widehat{x}_{3} ; \quad z_{2}=\lambda_{n-2}\left\lceil x_{1}-\widehat{x}_{1}\right\rfloor^{(n-2) / n} \\
\vdots \\
\dot{\hat{x}}_{n}=z_{n} ; \quad z_{n}=\lambda_{0}\left\lceil x_{1}-\widehat{x}_{1}\right\rfloor^{0},
\end{gathered}
$$

where $\lceil\xi]^{\alpha}=|\xi|^{\alpha} \operatorname{sign}(\xi)$, and gains $\left\{\lambda_{j}\right\}_{j=1}^{n}$ with $\lambda_{j}>0$. Note that differentiator (13) preserves the structure of the plant using dynamic extensions $z_{j}=\lambda_{n-j}\left\lceil x_{1}-\widehat{x}_{1}\right\rfloor^{(n-j) / n}$ that inject the estimation error in the differentiator dynamics. To see this, consider the estimation error $\widetilde{x}_{j}=x_{j}-\widehat{x}_{j}$ for $j=1, \ldots, n$. Then, one obtains the estimation error dynamics:

$$
\begin{gathered}
\dot{\tilde{x}}_{1}=-\lambda_{n-1}\left\lceil x_{1}-\widehat{x}_{1}\right\rfloor^{(n-1) / n}+\tilde{x}_{2}, \\
\dot{\tilde{x}}_{2}=-\lambda_{n-2}\left\lceil x_{1}-\widehat{x}_{1}\right\rfloor^{(n-2) / n}+\tilde{x}_{3}, \\
\vdots \\
\dot{\tilde{x}}_{n}=-\lambda_{0}\left\lceil x_{1}-\widehat{x}_{1}\right\rfloor^{0}+u+\varphi .
\end{gathered}
$$

Therefore, an adequate tuning of $\left\{\lambda_{j}\right\}_{j=1}^{n}$ produces $\tilde{x}_{j}=x_{j}-$ $\widehat{x}_{j}=0$, for any $j=1, \ldots, n$, after some finite-time $t_{f d}$, [33], p. 230. Finally, at this point, since $\widehat{x}_{1} \in \mathscr{C}^{n}$, then $\widehat{x}_{j}=x_{j}$ is exact for any $t \geq t_{f d}$.
3.3. Disturbance Observer Based on Fractional Differintegral Sliding Modes. The objective at this point is reject, exactly and in finite-time, the Hölder disturbance by means of the control $u$ for system (7). To this end, let control input be

$$
u=u_{0}+u_{s}
$$

where the continuous nominal control $u_{0}$ stabilizes the ideal system without disturbance [10], and the differintegral sliding mode control $u_{s}$ compensates the matched Hölder disturbance $\varphi$ [26-28]. Let $\sigma$ be a penalization variable that measures the deviation from the ideal system (without disturbances) to the real system defined by (7). Ideally, that is, assuming full access to the state, the penalization variable would be defined by

$$
\sigma(t)=x_{n}(t)-x_{n}(0)-\int_{0}^{t} u_{0}(\tau) d \tau
$$

such that the enforcement and invariance of $\dot{\sigma}(t)=0$ imply $\dot{x}_{n}=u_{0}$, at which system (7) renders an ideal plant without disturbances. Nevertheless, since $x_{n}(t)$ is not measured, but estimated, the penalization variable $\sigma$ is instead defined as

$$
\sigma(t)=\widehat{x}_{n}(t)-\widehat{x}_{n}(0)-\int_{0}^{t} u_{0}(\tau) d \tau,
$$

which in virtue of the exact estimation of $x_{n}(t)$ by $\widehat{x}_{n}(t)$ becomes $\sigma=x_{n}(t)-\widehat{x}_{n}(0)-\int_{0}^{t} u_{0}(\tau) d \tau$ for $t \geq t_{f d}$, and consequently, $\dot{x}_{n}=u_{0}$; then, from $\dot{\sigma}=u_{s}+\varphi$, it is clear that $u_{s}=-\varphi$ for $\dot{\sigma}=0$; then, $\left(z_{n}\right)_{e q}=u_{0}$. Note that before $t_{f d}$ just the output $y=x_{1}$ is assumed to be known; nevertheless, the controller (depending on $\widehat{x}_{j} \rightarrow x_{j}$ as $t \rightarrow t_{f d}$ ) is being applied from initial conditions to assure its continuity for any $t \geq 0$; then, the stability is compromised by the convergence of the differentiator.

A naive choice to design $u_{s}$ to enforce $\sigma=0$ in finitetime would be either the first-order discontinuous control $u_{s}=-k_{s} \operatorname{sign}(\sigma), k_{s}>\sup |\varphi|$, or the higher-order continuous controller $u_{s}=-k_{s 1}|\sigma|^{1 / 2} \operatorname{sign}(\sigma)-k_{s 0} \int \operatorname{sign}(\sigma)$, with $k_{s 1}>$ $\sqrt{2 k_{s 0}}$ and $k_{s 0}>\sup |\dot{\varphi}|$, [24]; however, the former is discontinuous and the latter requires differentiability of the disturbance; then, both cases do not satisfy the problem statement. Thus, this motivates to look at the topological properties of fractional-order integral operators and in particular consider the following uniformly continuous fractional-order differintegral sliding mode control:

$$
u_{s}(t)=u_{s}\left(t_{r}\right)-k_{s t_{r}} I_{t}^{v} \operatorname{sign}(\sigma(t))
$$

with feedback gain $k_{s}>[\varphi]_{\mathscr{H}^{\nu}(\Omega)}$, and the differintegral order $v \in\left(0, v_{c}\right),\left\{t_{r}\right\}_{r \in \mathbb{N}_{0}}$ is the strictly increasing sequence of instants such that $\sigma\left(t_{r}\right)=0$, and consider $t_{0} \geq t_{f d}$ the first time at which $\sigma$ crosses zero at or after $t_{f d}$. We now have the following first main result.

Theorem 9. Consider system (20). For feedback gain $k_{s}>((3+$ $\nu) /(1-\nu))[\varphi]_{\mathscr{H}^{\nu}(\Omega)}$, there is a finite-time

$$
t_{f \varphi} \leq t_{0}+\frac{1}{\left(1-\mu^{1 / v}\right)}\left[\frac{\left|\dot{\sigma}\left(t_{0}\right)\right| \Gamma(\nu+2)}{k-[\varphi]_{\mathscr{C}^{\nu}(\Omega)}}\right]^{1 / v},
$$


such that $(\sigma(t), \dot{\sigma}(t))=(0,0) \forall t \geq t_{f \varphi}$, where $\mu=((k+$ $\left.\left.[\varphi]_{\mathscr{H}^{\nu}(\Omega)}\right) /\left(k-[\varphi]_{\mathscr{H}^{\nu}(\Omega)}\right)\right)(1+\nu)-1<1$.

Proof. The proof is built upon [34] and our previous proposals [28-30] in conjunction with the finite-time state estimator. Consider the fractional-order differintegral dynamics resulting from applying control (18) into (17):

$$
\dot{\sigma}(t)=\dot{\sigma}\left(t_{r}\right)-k_{s} t_{r} I_{t}^{v} \operatorname{sign}(\sigma)+{ }_{t_{r}} I_{t}^{\nu E} D_{r} D_{t}^{\nu} \varphi .
$$

whose existence of solutions has been studied in $[28,29,34]$. In accordance with the dynamic resetting memory principle [28-30], $\left\{t_{r}\right\}_{r=0}^{\infty}$ can be considered as an increasing sequence of time instants when $\sigma(t)$ crosses the zero value, that is, when $\sigma\left(t_{r}\right)=0$. In addition, by virtue of the continuity of $u_{s}(t)$, the value $u_{s}\left(t_{r}\right)$ can be approximated by its preceding value $u_{s}\left(t_{r}-T\right)$, where $T$ is the sampling time, during real-time digital implementation [28].

Thus, solving for an arbitrary time-interval $\left[t_{r}, t_{r+1}\right]$, with $r \in \mathbb{N}_{0}$, by applying the mathematical induction, one gets $\left|\dot{\sigma}\left(t_{r}\right)\right| \leq \mu^{r}\left|\dot{\sigma}\left(t_{0}\right)\right|$ for all $r \in \mathbb{N}_{0}$. In addition, the time of convergence is estimated as

$$
\left(t_{r+1}-t_{r}\right)^{\nu} \leq \mu^{r} \frac{\dot{\sigma}\left(t_{0}\right) \Gamma(\nu+2)}{k_{s}-[\varphi]_{\mathscr{H}^{v}(\Omega)}},
$$

leading to

$$
\begin{aligned}
t_{f \varphi}-t_{0} & =\sum_{r=0}^{\infty}\left(t_{r+1}-t_{r}\right) \\
& \leq \frac{1}{\left(1-\mu^{1 / v}\right)}\left[\frac{\left|\dot{\sigma}\left(t_{0}\right)\right| \Gamma(\nu+2)}{k-[\varphi]_{\mathscr{H}^{\nu}(\Omega)}}\right]^{1 / v},
\end{aligned}
$$

since the series is geometric. Thus, in virtue of continuity, $\dot{\sigma}(t)=0$ for all $t \geq t_{f \varphi}$. In addition, the Fundamental Theorem of Calculus implies $\sigma(t)=\int_{t_{f \varphi}}^{t} \dot{\sigma}(\tau) d \tau=0$ for all $t \geq t_{f \varphi}$.

Note that since control $u_{s}$ is continuous, the invariant $\dot{\sigma}(t)=0$ is exact, not just equivalent, enforced for any $t \geq t_{f \varphi}$. This produces, as a byproduct of such control strategy, an exact finite-time disturbance observer $u_{s}=-\varphi$, establishing the nominal system $\dot{x}_{n}=u_{0}$ in finite-time.

\subsection{Finite-Time Stabilization. Considering that, for $t \geq t_{f \varphi}$,} one obtains $\widehat{x}_{j}=x_{j}$ and $\dot{\hat{x}}_{n}=u_{0}$, the objective is the design of a nominal controller $u_{0}$ such that $x_{1}=0$ for any $t \geq t_{f x}$ for some finite-time $t_{f x}$. For that purpose, consider the following nominal control [10]:

$$
u_{0}=-\sum_{j=1}^{n} k_{j}\left[\widehat{x}_{j}\right]^{\alpha_{j}}
$$

such that the polynomial $s^{n}+k_{n} s^{n-1}+\cdots+k_{2} s+k_{1}=0$ is Hurwitz, and $\alpha_{j-1}=\alpha_{j} \alpha_{j+1} /\left(2 \alpha_{j+1}-\alpha_{j}\right)(j=2, \ldots, n)$, with $\alpha_{n+1}=1$ and $\alpha_{n}=\alpha$. The stability of the ideal system in closed-loop with the nominal control (23) is given in the following result of [10]:
Theorem 10 (see [10]). There exists $\epsilon \in(0,1)$ such that, for every $\alpha \in(1-\epsilon, 1)$, the origin $x=0$ is globally finite-time stable.

Remark 11. Using only output information, for system (7) subject to Hölder disturbances, the proposed combination of robust continuous control techniques, established in Theorems 9 and 10, enforces finite-time convergence, despite the fact that such disturbance lacks integer-order differentiability, usually required for robust continuous high-order sliding mode-based control.

\section{Simulations}

Simulations were programed with a sampling time step of $T=$ $0.1 \mathrm{~ms}$ based on the Euler integrator. The Grünwald-Letnikov method was considered to compute differintegrals based on the resetting memory principle.

Consider a dynamic system described by (7), of second order, that is, with $n=2$, subject to a Weierstrass-Mandelbrot type disturbance [14], see Figure 1(a):

$$
\varphi(t)=\cos (t)+\sum_{l=1}^{N} 10^{-0.7 \imath} \sin \left(10^{\iota} t\right)
$$

Note that, when $N \rightarrow \infty$, function $\varphi(t)$ is nowhere differentiable; nevertheless, such function possesses welldefined fractional derivatives for all orders strictly less than $v_{c}=0.7$. Thus, the order of fractional integration in the differintegral sliding mode control $u_{s}$ needs to comply with the condition $v<v_{c}=0.7$ established in Theorem 9 .

Gains of differentiator (13) are $\lambda_{1}=1.5 L^{1 / 2}$ and $\lambda_{0}=$ $1.1 L$, as suggested in [9], with $L=5$. The parameters of the differintegral sliding mode control are $k_{s}=5$ and $v=0.5$. The nominal control is simply $u_{0}=-4\left\lceil\hat{x}_{1}\right\rfloor^{1 / 3}-4\left\lceil\hat{x}_{2}\right\rfloor^{1 / 2}$.

Figure 1 highlights the simulation results of the application of the proposed method. Figure 1(b) shows the finitetime exact estimation $\tilde{x}_{1}=0$, while finite-time exact rejection of the Hölder disturbance is illustrated in Figure 1(c). The finite-time convergence of the state $x$ is shown in Figures 1(d), 1(f), and 1(e) highlighting the nominal and sliding modebased control signals, respectively.

It is worth remarking the fact that the theoretically continuous controller is able to enforce finite-time convergence in a system that is subject to Hölder continuous disturbances, using only the output information. Figure 1 shows an unavoidable high-frequency phenomena that is characteristic of any continuous controller implemented in discrete time, even more, considering nonsmooth dynamics of both differentiator and disturbance compensator. In order to clarify the preceding explanation, the same simulation is carried out considering full access to the state, whose results are shown in Figure 2, where a great improvement can be appreciated; nonetheless, in most of engineering application just part of the state is considered available for control purposes. 


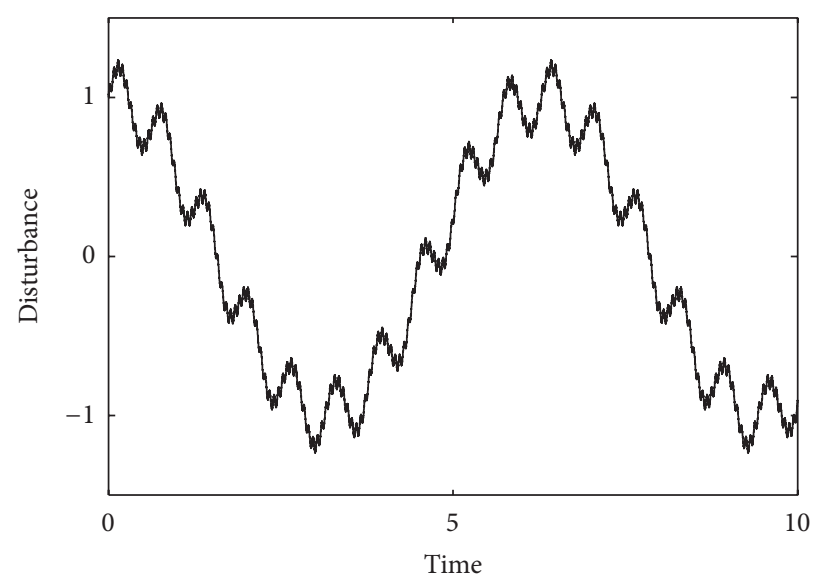

(a) Hölder disturbance $\varphi$

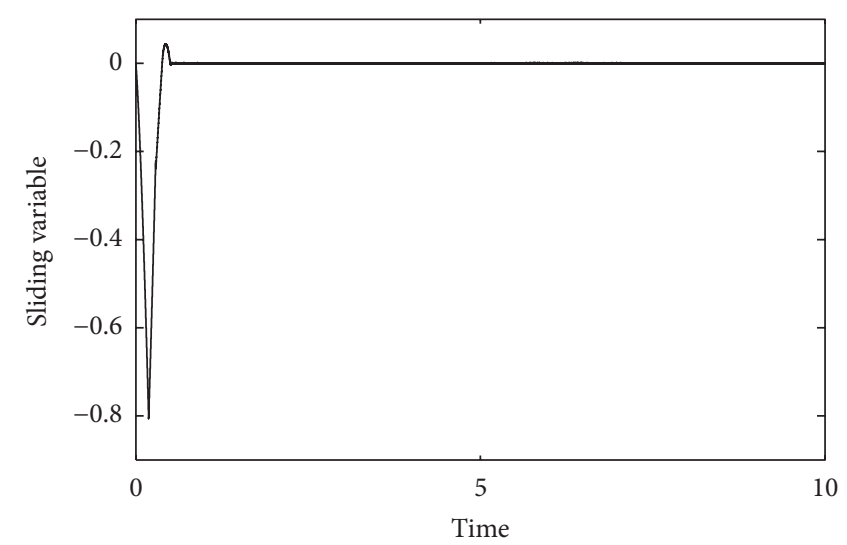

(c) Sliding variable $\sigma=\int\left(u_{s}+\varphi\right)$

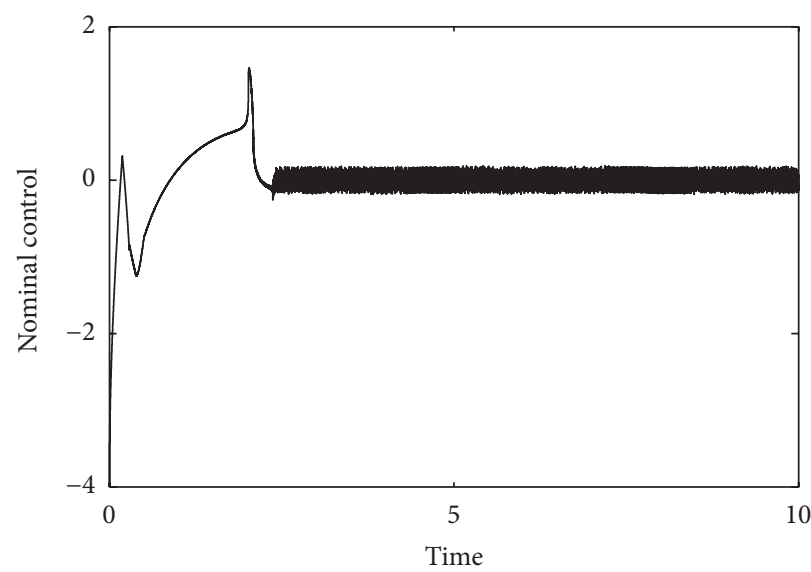

(e) Nominal control $u_{0}$

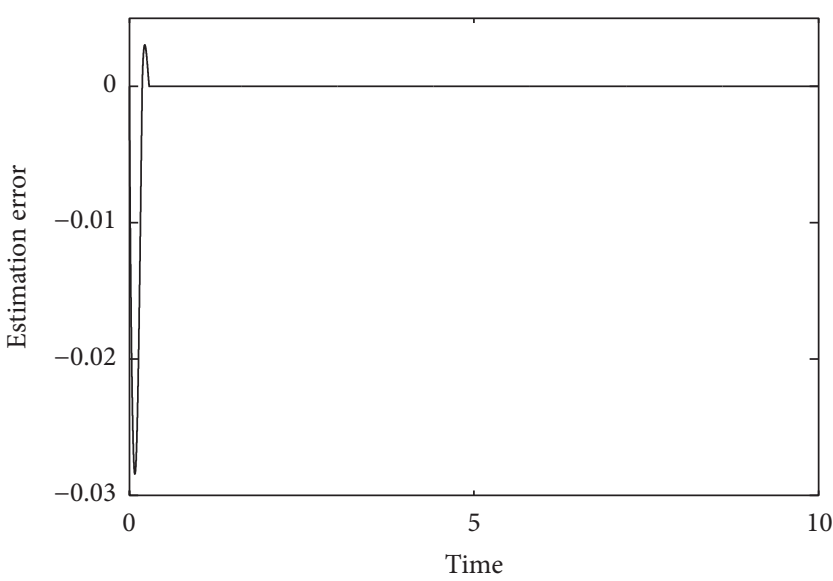

(b) Estimation error $\tilde{x}_{1}=x_{1}-\widehat{x}_{1}$

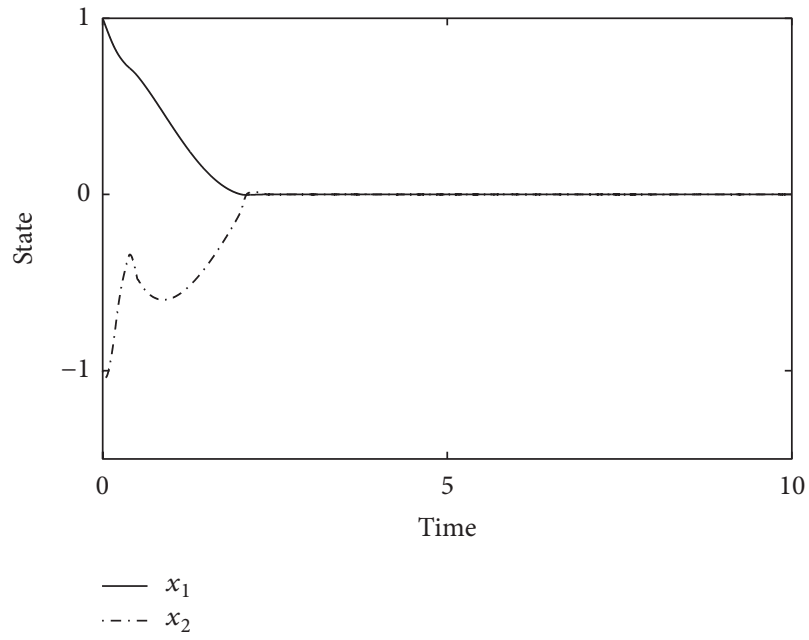

(d) State variables $x_{1}, x_{2}$

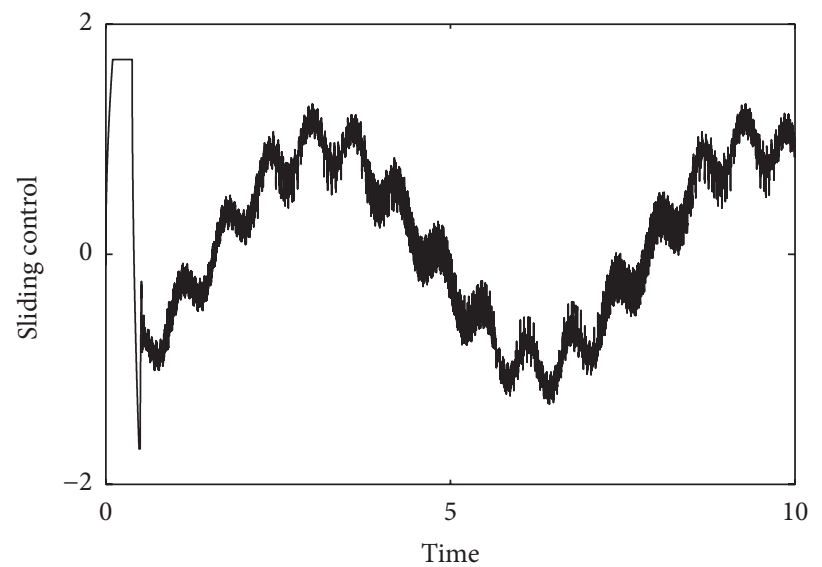

(f) Sliding control $u_{s}$

FIGURE 1: Simulation results. Finite-time output feedback control of a dynamic system subject to Hölder-type disturbances.

\section{Conclusions}

This paper proposes a synergistic combination of recent sound schemes for robust differentiation based on highorder sliding modes with fractional-order integral sliding modes to enforce finite-time stabilization of a class of dynamical systems subject to complex disturbance phenomena of Hölder type. Homogeneity is a key mathematical tool to design finite-time stabilizing controllers, providing robust differentiation and active disturbance compensation in finitetime. The resulting output feedback controller-estimator demonstrates the advantages of considering fractional-order 


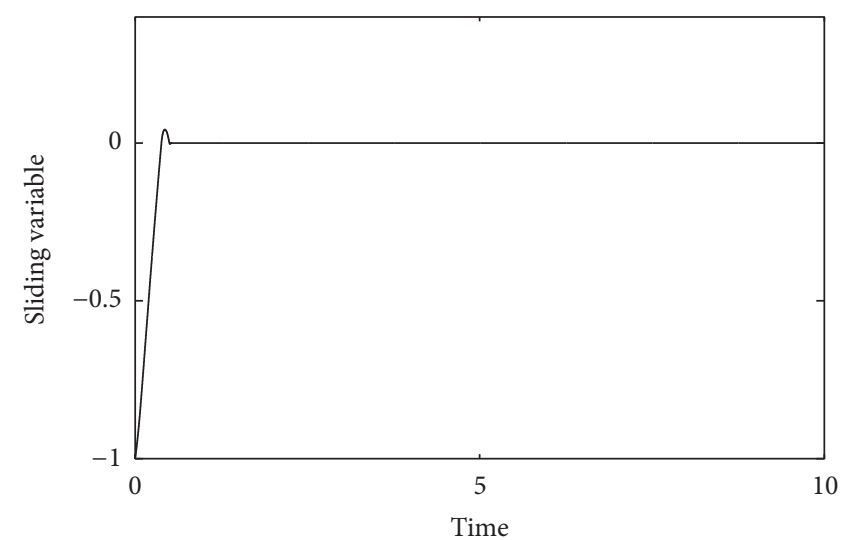

(a) Sliding variable $\sigma=\int\left(u_{s}+\varphi\right)$

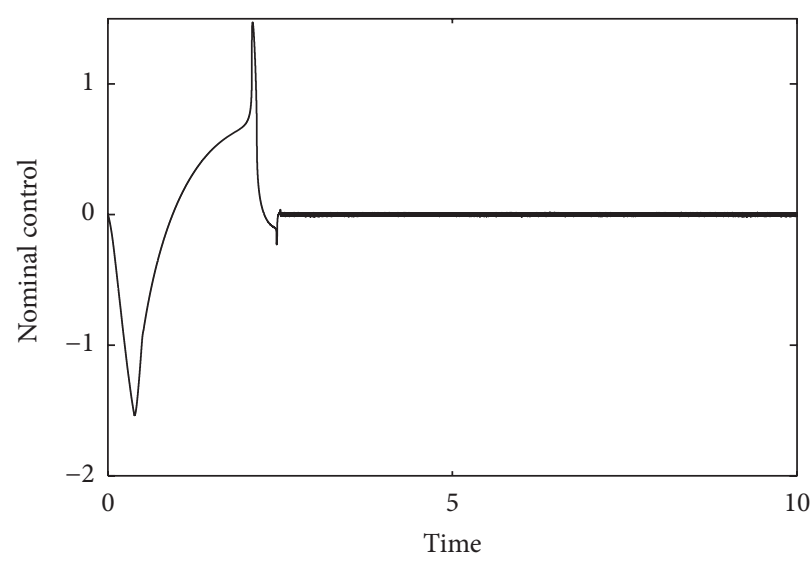

(c) Nominal control $u_{0}$

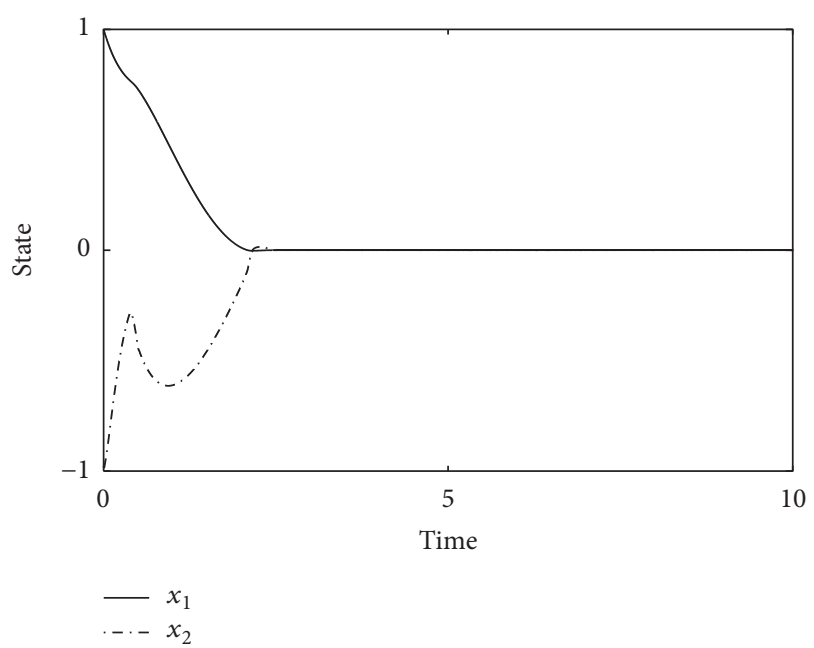

(b) State variables $x_{1}, x_{2}$

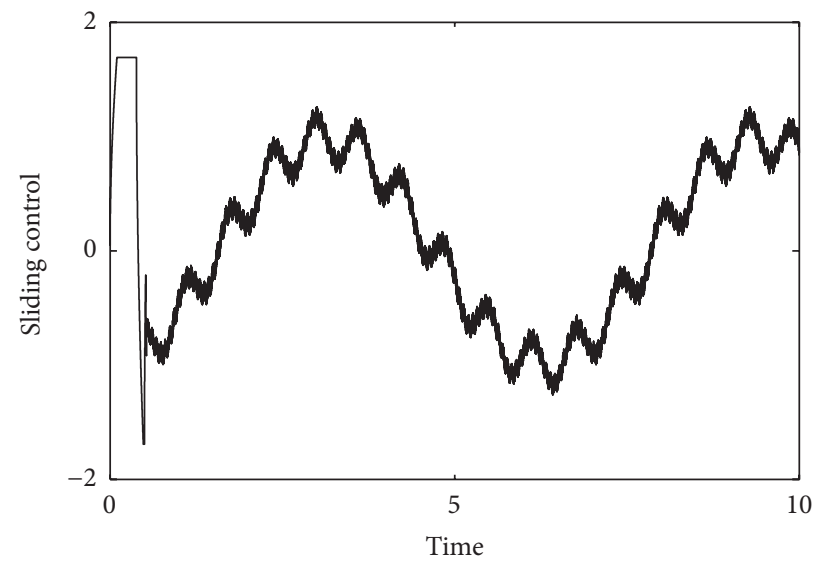

(d) Sliding control $u_{s}$

FIGURE 2: Simulation results. Finite-time state feedback control of a dynamic system subject to Hölder-type disturbances.

actions of control for a wider class of dynamic systems under most variate conditions of operation with potential applications in control of aerodynamic or underwater robots, as well as in contact robotic tasks with more diverse tribological interactions.

\section{Conflicts of Interest}

The authors declare that there are no conflicts of interest regarding the publication of this paper.

\section{References}

[1] S. P. Bhat and D. S. Bernstein, "Finite-time stability of homogeneous systems," in Proceedings of the American Control Conference, pp. 2513-2514, Albuquerque, NM, USA, June 1997.

[2] S. T. Venkataraman and S. Gulati, "Control of nonlinear systems using terminal sliding modes," Journal of Dynamic Systems, Measurement and Control, vol. 115, no. 3, pp. 554-560, 1993.

[3] M. Zak, "Terminal attractors for addressable memory in neural networks," Physics Letters A, vol. 133, no. 1-2, pp. 18-22, 1988.
[4] M. Zhihong, A. P. Paplinski, and H. R. Wu, "A robust MIMO terminal sliding mode control scheme for rigid robotic manipulators," Institute of Electrical and Electronics Engineers. Transactions on Automatic Control, vol. 39, no. 12, pp. 2464-2469, 1994.

[5] Y. Feng, X. Yu, and Z. Man, "Non-singular terminal sliding mode control of rigid manipulators," Automatica. A Journal of IFAC, the International Federation of Automatic Control, vol. 38, no. 12, pp. 2159-2167, 2002.

[6] V. Parra-Vega, A. Rodrguez-Angeles, and G. Hirzinger, "Perfect position/force tracking of robots with dynamical terminal sliding mode control," Journal of Robotic Systems, vol. 18, no. 9, pp. 517-532, 2001.

[7] S. P. Bhat and D. S. Bernstein, "Continuous finite-time stabilization of the translational and rotational double integrators," Institute of Electrical and Electronics Engineers. Transactions on Automatic Control, vol. 43, no. 5, pp. 678-682, 1998.

[8] S. P. Bhat and D. S. Bernstein, "Finite-time stability of continuous autonomous systems," SIAM Journal on Control and Optimization, vol. 38, no. 3, pp. 751-766, 2000.

[9] A. Levant, "Homogeneity approach to high-order sliding mode design," Automatica. A Journal of IFAC, the International Federation of Automatic Control, vol. 41, no. 5, pp. 823-830, 2005. 
[10] S. P. Bhat and D. S. Bernstein, "Geometric homogeneity with applications to finite-time stability," Mathematics of Control, Signals, and Systems, vol. 17, no. 2, pp. 101-127, 2005.

[11] C. Pukdeboon, "Finite-time second-order sliding mode controllers for spacecraft attitude tracking," Mathematical Problems in Engineering, vol. 2013, Article ID 930269, 2013.

[12] Z. He, C. Liu, Y. Zhan, H. Li, X. Huang, and Z. Zhang, "Nonsingular fast terminal sliding mode control with extended state observer and tracking differentiator for uncertain nonlinear systems," Mathematical Problems in Engineering, Article ID 639707, Art. ID 639707, 16 pages, 2014.

[13] Y. Hong, J. Huang, and Y. Xu, "On an output feedback finitetime stabilization problem," Institute of Electrical and Electronics Engineers. Transactions on Automatic Control, vol. 46, no. 2, pp. 305-309, 2001.

[14] B. Ross, S. G. Samko, and E. R. Love, "Functions that have no first order derivative might have fractional derivatives of all orders less than one," Real Analysis Exchange, vol. 20, no. 1, pp. 140-157, 1994/95.

[15] J. A. C. Humphrey, C. A. Schuler, and B. Rubinsky, "On the use of the Weierstrass-Mandelbrot function to describe the fractal component of turbulent velocity," Fluid Dynamics Research, vol. 9, no. 1-3, pp. 81-95, 1992.

[16] J. A. T. Machado, "Fractional order modelling of dynamic backlash," Mechatronics, vol. 23, no. 7, pp. 741-745, 2013.

[17] S. G. Samko, A. A. Kilbas, and O. I. Marichev, Fractional Integrals and Derivatives, Theory and Applications, Gordon and Breach, Yverdon, Switzerland, 1993.

[18] I. Podlubny, Fractional Differential Equations, vol. 198 of Mathematics in Science and Engineering, Academic Press, San Diego, Calif, USA, 1999.

[19] J. F. Gomez-Aguilar, H. Yepez-Martinez, C. Calderon-Ramon, I. Cruz-Orduña, R. F. Escobar-Jiménez, and V. H. OlivaresPeregrino, "Modeling of a mass-spring-damper system by fractional derivatives with and without a singular kernel," Entropy, vol. 17, no. 9, pp. 6289-6303, 2015.

[20] C. G. Merrett and H. H. Hilton, "Fractional order derivative aero-servo-viscoelasticity," International Journal of Dynamics and Control, vol. 5, no. 2, pp. 239-251, 2017.

[21] X.-H. Chang, J. Xiong, and J. H. Park, "Fuzzy robust dynamic output feedback control of nonlinear systems with linear fractional parametric uncertainties," Applied Mathematics and Computation, vol. 291, pp. 213-225, 2016.

[22] Y. Wang, J. Chen, and L. Gu, "Output feedback fractionalorder nonsingular terminal sliding mode control of underwater remotely operated vehicles," Scientific World Journal, vol. 2014, Article ID 838019, 2014.

[23] Y. Wei, P. W. Tse, Z. Yao, and Y. Wang, "The output feedback control synthesis for a class of singular fractional order systems," ISA Transactions, vol. 69, pp. 1-9, 2017.

[24] A. Chalanga, S. Kamal, and B. Bandyopadhyay, "A new algorithm for continuous sliding mode control with implementation to industrial emulator setup," IEEE/ASME Transactions on Mechatronics, vol. 20, no. 5, pp. 2194-2204, 2015.

[25] A. Levant, "Robust exact differentiation via sliding mode technique," Automatica. A Journal of IFAC, the International Federation of Automatic Control, vol. 34, no. 3, pp. 379-384, 1998.

[26] A.-J. Muñoz-Vázquez, V. Parra-Vega, and A. Sánchez-Orta, "Uniformly continuous differintegral sliding mode control of nonlinear systems subject to Hölder disturbances," Automatica.
A Journal of IFAC, the International Federation of Automatic Control, vol. 66, pp. 179-184, 2016.

[27] A.-J. Muñoz-Vázquez, V. Parra-Vega, and A. Sánchez-Orta, "Fractional integral sliding modes for robust tracking of nonlinear systems," Nonlinear Dynamics. An International Journal of Nonlinear Dynamics and Chaos in Engineering Systems, vol. 87, no. 2, pp. 895-901, 2017.

[28] A. J. Muñoz-Vázquez, V. Parra-Vega, and A. Sánchez-Orta, "Continuous fractional sliding mode-like control for exact rejection of non-differentiable Hölder disturbances," IMA Journal of Mathematical Control and Information, vol. 34, no. 2, pp. 597-610, 2017.

[29] A. J. Muñoz-Vázquez, V. Parra-Vega, and A. Sánchez-Orta, "Continuous fractional-order sliding PI control for nonlinear systems subject to non-differentiable disturbances," Asian Journal of Control, vol. 19, no. 1, pp. 279-288, 2017.

[30] A.-J. Muñoz-Vázquez, H. Ramírez-Rodríguez, V. Parra-Vega, and A. Sánchez-Orta, "Fractional sliding mode control of underwater ROVs subject to non-differentiable disturbances," International Journal of Control, Automation and Systems, vol. 15, no. 3, pp. 1314-1321, 2017.

[31] C. Izaguirre-Espinosa, A. J. Muñoz-Vázquez, A. Sánchez-Orta, V. Parra-Vega, and P. Castillo, "Attitude control of quadrotors based on fractional sliding modes: theory and experiments," IET Control Theory \& Applications, vol. 10, no. 7, pp. 825-832, 2016.

[32] A. Isidori, "Nonlinear Control Systems," in Communications and Control Engineering, Springer-Verlag, London, UK, 3rd edition, 1995.

[33] Y. Shtessel, C. Edwards, L. Fridman, and A. Levant, Sliding Mode Control and Observation, Birkhäuser, Springer, New York, NY, USA, 2014.

[34] R. Garrappa, "On some generalizations of the implicit Euler method for discontinuous fractional differential equations," Mathematics and Computers in Simulation, vol. 95, pp. 213-228, 2014. 


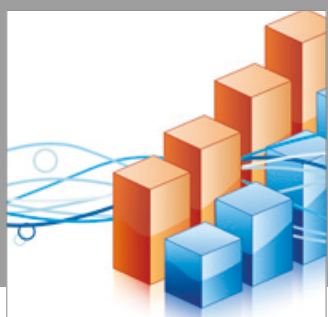

Advances in

Operations Research

vatersals

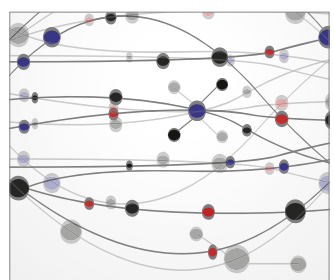

\section{The Scientific} World Journal
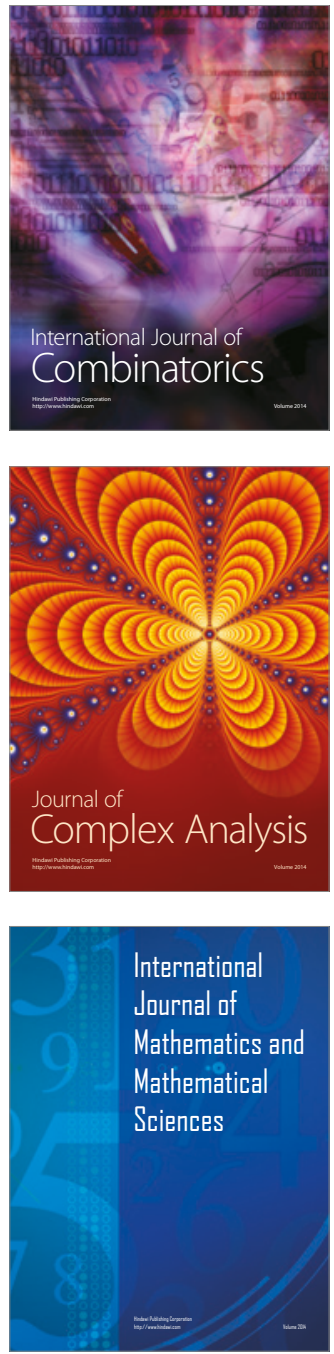
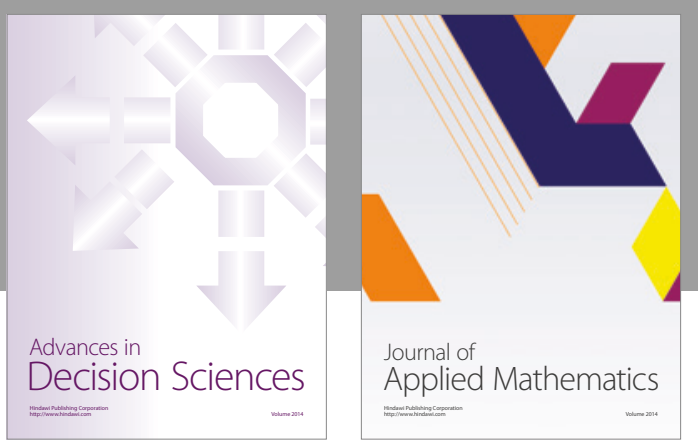

Algebra

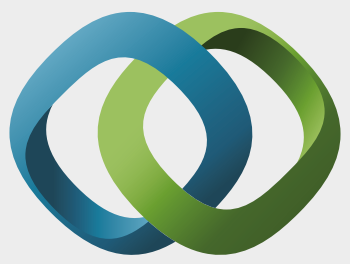

\section{Hindawi}

Submit your manuscripts at

https://www.hindawi.com
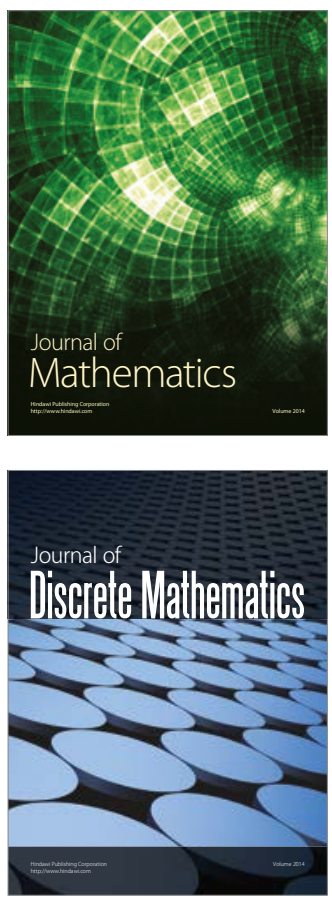

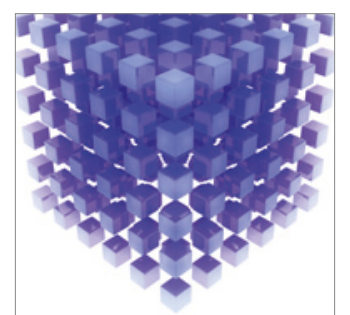

Mathematical Problems in Engineering
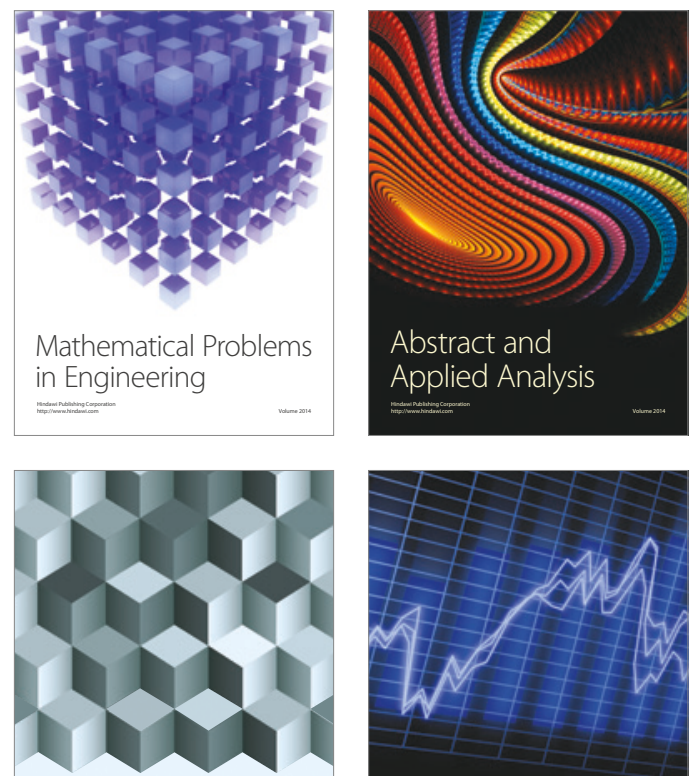

Journal of

Function Spaces

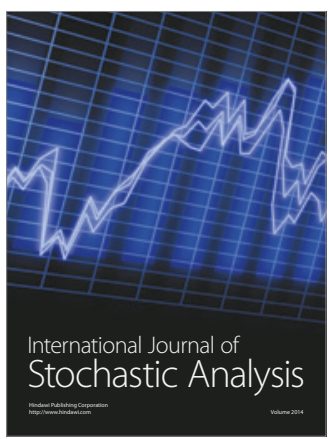

Probability and Statistics
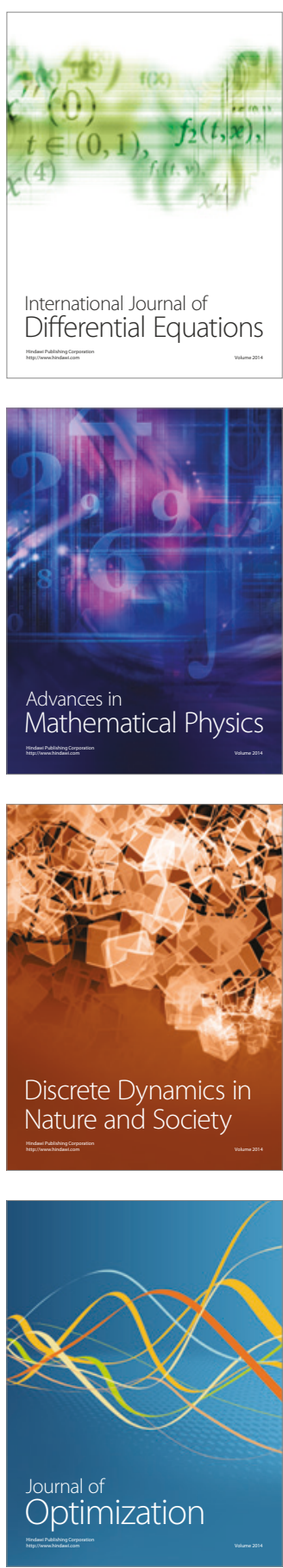